\title{
Sensing the World: Challenges on WSNs
}

\author{
Válter Rocha \\ Instituto Agilus de Inovação em Tecnologia de Informação, S.A. \\ Rua Dr. Afonso Cordeiro 877, sala 202 \\ 4450-007 Matosinhos \\ Portugal \\ valter.rocha@iaiti.pt \\ FEUP - Faculdade de Engenharia da Universidade do Porto \\ valter.rocha@fe.up.pt
}

\begin{abstract}
Wireless sensor network (WSNs) is a demanding multidisciplinary field that has been target of research in the last decade. The increasing demand for security and automated monitoring of things and places makes WSNs a promising technology although there are still many open research contributions needed to make this the standard of environmental sensing.

The goal of this article is to identify the research challenges on WSNs by dividing them into functional groups, building on previous work. We followed a structured approach based on a simplified yet complete vision of a design space for WSNs. Moreover, this work aims to identify research gaps and investigation fields yet unexplored or hardly explored by researchers in order to plot paths for future research. Several challenges and research areas were identified, like Models for Sensor Networks, Benchmarking Methodologies, Distributed Processing, Interface WSNs and Network Reprogramming.

Some of these challenges will become even clearer as increasingly WSNs become Wireless Sensor and Actuator Networks (WSANs).
\end{abstract}

Key Words: Wireless Sensor Networks, WSN: Pervasive Networking, Mesh Networking.

\section{Introduction}

Embedded devices have been part of our lives for the last 50 years but just recently the advances in semi-conductor technology, in low power wireless radios and MEMS (Micro-Electro-Mechanical Systems) technologies have enabled the production of cost effective, low-power, and small scale devices. These inexpensive, low-power communication devices can be deployed throughout a physical space, providing sensing, processing and communicating capabilities.

WSNs are the breakthrough approach based on networks of devices that can be densely deployed in human aggressive and inaccessible environments, to sense and instrument the environment and monitor with high accuracy physical phenomena. Each one of these devices is called a sensor node. Each node should not be larger than a few square millimeters and its target cost is less than US\$1.00, including radio, mi- 
crocontroller, power supply and sensor (capable of sensing temperature, light, vibration, sound, etc.).

To realize the opportunities created by this concept, information technology must address a new collection of challenges. The individual nodes in a WSN are inherently resource constrained: They have limited processing speed, storage capacity, and communication bandwidth. These devices have substantial processing capability in the aggregate, but not individually, so we must be able to combine their many capabilities within the network itself. Due to the multidisciplinary nature of WSNs, these challenges are somehow disperse. Consequently each study on the topic presents a view on existing challenges and difficulties present in a certain field.

In order to present these challenges in a systematic way, a structured approach was followed. This approach was based on the work presented in [1] where the need for standardization in order to coordinate efforts and encourage developments in this area is discussed.

This paper is organized as follows. We start by describing the Design Space in Section 2, continue by presenting some possible applications on WSNs in Section 3. On sections 4 and 5 we discuss the architecture of a single node and an entire network. Section 6 presents technology gaps and their respective research paths. Finally we discuss the conclusions and future work in section 7.

\section{Design Space}

Although WSNs share common problems and solutions with embedded systems and ad hoc networks in what concerns hardware and network issues, there are new challenges that rise from the fact that these devices are resource constrained.

Several researchers approach the design of WSNs on an application oriented basis while others, like for example Römer and Mattern [1], structure such challenges in classes and name this structure "The Design Space of Wireless Sensor Networks". Based on this work, we propose our simplified yet complete vision of the design space by refactoring the one proposed in [1]. Some proposed design space dimensions like deployment, coverage and network size or like network topology and connectivity relate to the same problem influencing each other, therefore should not be dissociated. Others like Application requirements and Environment interaction that relate to the application specific needs should be introduced to further improve the design concepts on WSNs.

\subsection{Application requirements and Environment interaction}

Application specific requirements define almost every other topic in WSNs. The large amount of heterogeneous sensor nodes can be used freely to create many different solutions. There is no such thing as a general solution still research should keep generalization in mind.

As WSNs are environmental event-driven, their activity graph can vary a lot during time. While most of the time activity is very low, when some environmental change occurs events come in bursts and can generate traffic problems. 


\subsection{Network Dynamics}

The deployment and mobility of nodes affect the network topology as there is uncertainty on the nodes location and density. Moreover, some nodes can be deployed or arrive to a specific network, attached to an object or by their own means, becoming active members and making deployment a continuous process. This forces the network protocols to be flexible and dynamic in order to react to the different demands of applications. Also the degree and speed of movement can influence in the time the nodes are available and therefore their usability in the network.

\subsection{Cost, Size, Resources, Energy and Lifetime}

The application design requirements determine the size, cost and energy specifications. While some applications may need thousands of simple nodes, highly resource constrained, others could require just some specialized and complex nodes or a mix of the two solutions. Similarly the price of each node can vary from be as low as US $\$ 1.00$ or reach US\$1000.

Energy resources, and consequently the WSN lifetime, are directly dependant on cost and size factors as the size of the batteries or harvesting devices is directly proportional to the amount of energy they accumulate or generate. Also computing power and storage depend on the price, although currently this is not such a relevant issue because used Microcontroller Units and flash storage chips are at a very low price.

The amount of resources available at the nodes is also a determinant factor in the degree of complexity of each sensor node's embedded software and possibly with the entire network's routing and processing capabilities.

\subsection{Heterogeneity and Complexity}

Sensor networks can be deployed in practically any scenario and may require many types of distinct sensor nodes to accomplish the expected results. Differences in nodes range from computing power to size, sensor specialization or mobility.

The more heterogeneous the network is the more powerful and generic it becomes. But on the other hand, more robust and complex routing and communications protocols it requires.

Heterogeneous networks may use nodes with high processing capabilities or high energy resources to distribute processing efforts and power usage amongst the network nodes.

Typical configurations use a more powerful specialized node (sink node) to transmit data generated in the network to a computer source via internet, GPRS/UMTS or satellite, and possibly providing position by means of a GPS device.

\subsection{Infrastructure and Communication Modality}

As infrastructures are very expensive and sometimes impossible to deploy, Ad Hoc networks are becoming the standard in WSNs. While in the first case, communications are made via the base station in the second nodes communicate with each other directly without the need for an infrastructure. Nodes usually serve as routers, forwarding messages hop-by-hop on behalf of other nodes.

In both cases several communication modalities can be used: radio, diffuse light, laser, inductive and capacitive coupling or sound. The most common is radio trans- 
mission because it doesn't need line of sight which is a requirement in many applications.

\subsection{Network Topology and connectivity}

The application requirements play an important role in the Network Topology by defining either if the sensor coverage (area of interest covered by sensor nodes) should be dense or sparse, if the network should be more or less dynamic and the deployment area wide or narrow. A dense coverage is used if more accuracy and redundancy is required. The redundant sensor nodes could be used to make the network more robust replacing "dead" sensors or managing power savings between sensors by entering sleep mode. If the application requires mobility or a random deployment of some sensor nodes, the protocols have to be more dynamic to cope with the changes experienced in the network.

The Network Topology depends on its Diameter (max number of hops between any two nodes) and size (number of nodes) determined by the specific requirements.

The simplest topology is the single-hop network where every sensor can communicate directly to all the other sensors. A most elaborated topology is needed when nodes can't reach at least one node. To be able to communicate to a non reachable node, other nodes are used as routers. This is called multihop networking. In some applications on top of the arbitrary graph generated by the multihop network there is a simpler overlay structure that forms an organized tree or set of connected stars.

Data routing, distributed processing, latency and robustness are affected by the topology of the network.

The topology of the network and communications hardware ranges can influence the connectivity of the network. If every node can reach any node, the network is connected. If the nodes can are arranged in partitions and a mobile node provides communication occasionally, the communication is called sporadic. Communications protocols are influenced by connectivity.

\subsection{Quality of Service (QoS)}

QoS refers to the capability of a network to provide better service to selected network traffic over various technologies. A sensor network should provide the necessary quality of service to cope with the application needs. For example, if the application requires real-time access to data the network should be designed to support such feature. Minimal QOS requires that the network should always be operative and immune to: failures (robustness), deliberate attacks (tamper-resistance), Communications eavesdropping, and detection (unobtrusiveness or stealth). Moreover the network should provide the means to control and efficiently use network resources.

\section{Applications for sensor networks}

Such an enabling technology provides means for many new types of applications to become implementable. As described in [2], applications can be classified in three classes: i) monitoring space (environmental and habitat monitoring); ii) monitoring things (precision agriculture, indoor climate control, surveillance, treaty verification, 
and intelligent alarms); and iii) monitoring space and things (the interactions of things with each other and the encompassing space, the most complex interactions, including monitoring wildlife habitats, disaster management, emergency response, ubiquitous computing environments, asset tracking, healthcare, and manufacturing process flow).

Research projects that involve WSNs are on their way for military applications, environmental observation, intruder surveillance, bridge and tunnels structure monitoring, civil protection applications and many others. Examples on research activities are the system for bird observation on Great Duck Island [3], ZEBRANET [4], Glacier Monitoring [5], Cattle Herding [6], Bathymetry [7], Ocean Water Monitoring [8], Grape Monitoring [9], Cold Chain Management [10], Rescue of Avalanche Victims [11], Vital Sign Monitoring [12], Power Monitoring [13], Parts Assembly [14], Tracking Military Vehicles [15], Self-Healing Mine Field [16], Sniper Localization [17], Early Warning Fire Detection [18].

WSNs need to be designed to accomplish low cost, low power solutions. Both hardware platforms and network protocols have to be well suited to the intrinsic constraints of this new technology and, in the near future, when the cost and size problems are overcome, many other applications will become feasible.

\section{Node Architecture}

\subsection{Hardware design}

A typical hardware configuration for a wireless sensor node is composed of a microcontroller, data storage, radio / optical / ultra-sound transceiver, power supply and sensors.

The Micro-Controller Unit (MCU) is usually an Atmel or Texas Instruments, with analog-to-digital converters (ADCs), and SPI, I2C, as I/O inputs to interface with sensors and actuators. Some other important issues like chip computing power, data storage, size and cost, power consumption in awake and sleep modes and in transitions between the modes are decisive factors for choosing the MCU.

The size and low power constraints limit the amount of the on-chip storage. Typically MCUs reach 10 Kbytes of RAM for data and less than 100 Kbytes of ROM for program storage. However, small size Flash memory is getting cheaper and provides large amount of data storage for a low cost. Still the power consumption of such devices reaches $100 \mathrm{~mW}$ or even more when in high speed write procedure so this has to be taken into account when designing the node.

Infineon or Chipcon radio devices are usually used in WSNs. The digital modulation techniques more common are amplitude-shift-keying (ASK) and frequency-shiftkeying (FSK) although some researchers [19] are testing other modulation methods like On-Off-Keying (OOK).

Some nodes don't need to wake up much often because of the nature of measurements they have to make, but as they have to be in touch with the network they need to wake up to receive incoming messages. An important step towards low power communications would be the wake-up on radio transmissions. This way, nodes could save power by sleeping and when a message arrives, they wake up and respond. 
The need of monitoring the world is the reason of the existence of WSNs and transducers play a great role on electrically representing physical phenomena. There are several types of sensors nowadays. While piezoelectric sensors are usually very expensive and big, MEMS transducers provide good accuracy for low cost and small size boosting the miniaturization of sensor nodes.

Every aspect of the WSNs' design has to take into account the power limitations and needs as this is certainly one of the most important issues in the WSNs' concept. As the deployment of sensor nodes mostly occurs in inaccessible places, power sources have to be designed to their network node's expected lifetime.

MCUs are getting smaller and more power efficient per clock frequency operating now at about1mW@10MHz and 1uW@ @leep. Solar panels generate 10mW/ $\mathrm{cm}^{2}$ indoors and their internal peripherals like ADCs are now produced for low power consumptions.

\subsection{Embedded Software}

In a highly complexity technology such as WSNs, Operating Systems (OS) play an important role in solving many important design issues regarding hardware abstraction and resource management. OSs designed for WSNs are quite different from the ones used in traditional embedded systems mainly because of the power, processing and storage constraints that require power efficiency, reactivity, mobility, faulttolerance, and concurrency.

There are two main types of OSs used in WSNs: Multithread-Driven is the traditional embedded systems approach adapted to WSN issues, where each task is given a CPU slot and all tasks concur to access resources; Event-Driven responds to events such as an incoming data packet or a sensor reading, by calling an event handler and allocating CPU and resources to solve the event in hands to completion. Event-Driven OS are best suited to address the requirements of WSNs because of the event intrinsic nature of WSNs.

Some examples of multithread OSs are RETOS [20] and MANTIS-OS [21]. The mostly used Event-Driven OS are TinyOS [22], SOS [23], Contiki [24], and Yatos [25]. Although Contiki runs an Event-Driven kernel, it introduces a new concept named protothreads that provides a linear, thread-like programming style on top of the kernel.

Another important concept when choosing an OS to run on WSNs is the support for safe and efficient re-programming of nodes. This is already addressed in some OSs like TinyOs, SOS and a virtual Machine for TinyOS named Maté [26] that allow a complete reprogramming of sensor nodes. Furthermore SOS and Maté allow efficient reprogramming of parts of the code by uploading the corresponding binary file.

Other issues like battery management, peripheral support and communications stack should be embedded in OS features.

\section{Network Architecture}

The concept of WSNs implies many different aspects that should be optimized for better power saving, performance and efficiency. Each application can demand differ- 
ent network topologies and different deployment densities. This impacts the communications and routing protocols design.

\subsection{Physical, MAC, and Link layers}

The design of the physical layer could improve significantly the energy efficiency of the network. Some factors like overhead, redundancy, etc. should be looked at when designing the physical layer. Some work on this topic can be found in [27] and [28].

Media Access Control (MAC) is one of the most active research areas in WSNs. The main research topic is related to keeping the nodes in power saving mode as much time as possible. Consequently, most of the work is closely related to TDMA.

Some work has been done regarding packet size, energy efficiency, Forward Error Correction (FEC) and transmission power variation on the energy spent per useful bit. In [29], the authors mention that there is work relating to "taking into account the degree of redundancy that an aggregated message carries on the link layer, which is much more specific to the situation in wireless sensor networks". However there is already some work done in this field.

Although most of the concepts related to addressing techniques are similar to Ad Hoc Networks, there are also specific problems. For example, geographic addressing nodes could be very helpful for routing algorithms. A new interesting concept is content-based addresses that seem very intuitive in WSNs because of their data-oriented nature.

\subsection{Synchronization and Localization}

In some applications the data acquired in all nodes makes sense as a whole and therefore needs to be synchronized. This is not as trivial as it could appear because there are delays in transmissions and there is no broadcasting clock to synchronize nodes. This is a much interesting research area for such applications. A good work in this area is [30].

The localization of sensor nodes using just the relative positions of the sensors is a very important and researched area in which many approaches have been made such as exploiting received signal strength indicators, time of arrival, time difference of arrival, or angle of arrival. Distributed algorithms are playing a great role in increasing precision.

\subsection{Topology and Network Layer}

When the number of nodes in the range of a particular node is big, a traditional flooding-based routing could quickly reach enormous amounts of repetition of messages. Two very common approaches to address this issue are transmission power control and clustering. Some other approaches stated in [31] include:

- Small Minimum Energy Communication Network: Creates a sub-graph of the sensor network that contains the minimum energy path.

- Gossiping: Sends data to one randomly selected neighbor. Avoids implosion problem but message propagation takes longer time.

- SPIN: Whenever a node has available data, it broadcasts a description of the data and sends it only to the sensor nodes that express interest. 
- SAR: Creates multiple trees where the root of each tree is one hop neighbor from the sink. A sensor node selects a tree for data to be routed back to the sink according to the energy resources and additive QoS metric.

- LEACH: Forms a two level cluster hierarchy, where cluster members send data to the cluster head which in turn sends it to the base station. Energy dissipation is evenly spread by dissolving clusters at regular intervals and randomly choosing the cluster heads.

- Directed Diffusion: A sink sends out an interest which propagates in the network and sets up gradients for data to flow from source to sink.

The network layer is the most active research area after MAC and topology control. Some Ad Hoc solutions could serve as an inspiration to developers in this field, nevertheless there are specific issues regarding only WSNs (scalability, energy efficiency and data-centricness). The traditional routing algorithms unicast, multicast, anycast, and convergecast are still used in WSNs. New approaches designed specifically for WSNs, like geographic and data-centric routing, are also explained in this section.

Unicast routing protocols is a very well covered area in Ad Hoc Networks. The main contribution of this algorithm is the way it deals with energy as a scarce resource. It takes in consideration battery power in nodes to calculate the best path. A good example of unicast protocol is LEACH. However other protocols that disregard power may perform more efficiently in some circumstances.

Multicast is similar to unicast. Energy-efficiency problem is once again covered by this protocol. One emerging approach is stochastically constrained multicast, where just a certain percentage of nodes answers when a request is made. The objective is to rotate sleeping patterns of nodes. This can harmonize application requirements with lower layer behavior.

Anycast relates to sending messages to an object name that is multiple instantiated in the network. Typically the closest one is used. This is commonly useful for service discovery which is still a much unexplored area in WSNs.

Convergecast consists on collecting data from several nodes in a central point (Sink). This is an important concept in WSNs as it is very close to in-networking processing and aggregation concepts.

Geographic routing is defined as directing a packet not to a node but to a target area instead. Any node present in the area is a candidate destination node and can receive and process a message. This has an obvious importance for WSNs because of the dynamics of the networks and the environmental-driven monitoring.

Data-centric routing is perhaps the core abstraction of WSNs. It combines the applications need to access data by using a natural framework for in-network processing. When a sensor node has new readings publishes these values. Interested nodes can subscribe to such events. One of the most popular and more cited publications in this area is "directed diffusion" [32], even though some of its performance and functional characteristics are not entirely understood or explained. Another recent approach is similar to pear-to-pear data storage in the internet, but this is still not thoroughly investigated. 


\subsection{Transport}

The transport protocol, just recently attracted the attention of researchers. This layer addresses mainly the issues of QoS versus the amount of energy needed to provide such service a good work can be found on [34].

\section{Technology Gaps and Research Opportunities}

As this is a novel technological area there are still many roads ahead and many gaps that can be filled. At the writing of this survey some important issues still lack an efficient approach and are therefore open for contributions from researchers with interest in this field. Some of these topics that have to be addressed are here mentioned as a starting point to researchers interested in this technology.

\subsection{Models for Sensor Networks}

Some efforts have been made to systematize common problems and main challenges. A good approach was made in [1] to clarify the problems per topics, there are many possible approaches, as it was referred, and the model needs to be refined. There are questions that still need to be answered:

How can we classify networks based on applications and how does this influence on the network model?

How can we design the network to be energy efficient? What type of routing is best suitable for WSNs? Does the network model depend on each application?

Is there a general approach for models? Is there the need to design different algorithms to different network models? Is it possible to make distributed pre-processing?

\subsection{Benchmarking WSNs}

As referred before, each research group presents different views of models for WSNs relating to different parts of the design space. As there is no accepted standard model the lack of a clear and uniform view makes it very hard to evaluate protocols and compare them to each other.

After researchers reach one or more model representation suitable for some or all application classes, appropriate metrics for evaluating strengths and weaknesses of protocols need to be defined.

The next step is to develop an automatic procedure of classifying protocols using metrics defined, comparing performance to the ideal protocol and to the trivial one.

Such a platform would benefit the complete community providing means to benchmark research results and therefore helping specialists improve their one work.

\subsection{Error Detection and Correction algorithms}

WSNs are about gathering and distributing data. Communications are often in noisy and dynamic environments. There is still open space for the development of error detection and correction algorithms to shield communication from errors. 


\subsection{Security}

Security issues are always important when collecting and distributing data. Research in this field seams to derive from the ad hoc networks security solutions, still and as referred before, WSNs have specific problems that could introduce important security problems. There is clearly a technology gap that is open for researchers to fill.

\subsection{Distributed processing and storage algorithms}

WSNs are by definition distributed. The acquisition of data is done everywhere and deployed anywhere in the network. The resource constraints force the need to reduce the amount of communications and distribute data storage. Data acquired in a node could be pre-processed (filtered, compressed, aggregated, etc.) in that node to minimize transmissions, and processed as it flows through its path to the destination node. Some inspiration in peer-to-peer networks could be useful because of the similarities faced regarding distributed data storage problems. The need for distributed algorithms becomes even clearer as increasingly WSNs are becoming Wireless Sensor and Actuator Networks (WSANs). In cases where actuators have to react in conjunction to fulfill an objective there is the need to make the decision in a distributed way. The application of results from research in Social Networks could also be explored in what concerns decision making.

\subsection{Routing Protocols}

The purpose of WSNs is to harvest and distribute data. This has to be done in an efficient way especially as the deployment of such networks is usually made in difficult access places and the resources limit the network's lifetime. Good routing protocols are already in place although most of them assume static topologies and therefore some improvements could be useful.

A good approach would be a protocol that could predict the positioning of nodes by making a graph on the relative location of nodes to each other.

A good survey dedicated exclusively on routing protocols can be found in [33].

\subsection{Transport protocol}

At the time of this document some good transport protocols designed for WSNs are available, however there are still open issues regarding energy efficiency on transport protocols. One possible approach could be designing a protocol that reacts on energy, network topology and node location factors. Moreover the transport protocol should be able to provide transport of data between heterogeneous networks by allowing intermediate proxies. This would be useful both for WSNs and for traditional wireless networks.

\subsection{Interface WSNs}

Considerable amount of work has been done regarding WSN related issues yet interactions with the outside world and with other WSNs are still far from being completely explored.

One very interesting approach is made by the University of California at Berkley in the TinyDB [35] project. This approach faces the interface to WSNs as if they were a database. The main issue is that the SQL queries are not optimized for energy savings. 
A possible approach is to design energy-efficient query language to take full advantage of the WSNs as referred in [36], [37], and [38]. Other possible approach is to integrate node location and network topology inputs in the database to allow appropriate routing of queries and information.

\subsection{Network Reprogramming}

Although operating systems already contemplate some aspects of network programming there is still space for research in this area. Some important issues on powerefficiency could still be improved and an enabling new concept of peer reprogramming could be explored. The idea behind this concept is very simple: Due to the heterogeneity of the networks and to the resource constraints of the program storage capabilities of the sensor nodes, although they might share the same hardware, some are specialized in specific tasks. In some cases it is interesting for a node to teach another node in range to do its work, when for example its battery is ending.

\section{Conclusions}

With the advances in processing power, sensor accuracy, miniaturization and production costs, WSNs will be used in a wider range of applications in the near future. Already deployed WSNs serve as a proof of concept, providing the means to monitor environmental data formerly impossible to collect due to the inaccessibility or characteristics of the environments where such physical phenomena take place.

Research on this field is very promising and has been attracting the scientific community for the last years. Some of the more active research areas are models for sensor networks, distributed processing, routing protocols, transport protocol and network reprogramming. Recent developments prove that there are many applications feasible with the available technology, assuring that in a near future WSNs will become commodities in what concerns sense and instrument the physical world. Furthermore WSNs are a factor of progress, enhancing productivity in sectors such as agriculture, transport and construction.

\section{References}

1. Kay Römer, Friedemann Mattern, "The Design Space of Wireless Sensor Networks", IEEE Wireless Communications, Vol. 11, No. 6, pp. 54-61, December 2004.

2. David Culler, Deborah Estrin, Mani Srivastava, "Overview of Sensor Networks “, Special Issue in Sensor Networks, IEEE Computer 37(8), Aug 2004: pp. 41-49.

3. Mainwaring et al., "Wireless Sensor Networks for Habitat Monitoring", WSNA, Atlanta, GA, USA, Sept. 2002.

4. P. Juang et al., "Energy-Efficient Computing for Wildlife Tracking: Design Tradeoffs and Early Experiences with ZebraNet", Proc. ASPLOS X, San Jose, CA, Oct. 2002.

5. K. Martinez et al., "GLACSWEB: A Sensor Web for Glaciers", Adjunct Proc. EWSN 2004, Berlin, Germany, Jan. 2004.

6. Z. Butler et al., "Networked Cows: Virtual Fences for Controlling Cows", WAMES 2004, Boston, MA, USA, June 2004. 
7. W. Marshall et al., "Self-Organizing Sensor Networks", UbiNet 2003, London, U.K., Sept. 2003.

8. “ARGO - Global Ocean Sensor Network”, http://www.argo.ucsd.edu, Jan. 2008

9. R. Beckwith, D. Teibel, and P. Bowen. "Pervasive Computing and Proactive Agriculture", Adjunct Proc. PERVASIVE 2004, Vienna, Austria, Apr. 2004.

10. R. Riem-Vis, "Cold Chain Management Using an Ultra Low Power Wireless Sensor Network", WAMES 2004, Boston, USA, June 2004.

11. F. Michahelles et al., "Applying Wearable Sensors to Avalanche Rescue", Computers and Graphics, vol. 27, no. 6, 2003, pp. 839-47.

12. H. Baldus, K. Klabunde, and G. Muesch. "Reliable Setup of Medical Body-Sensor Networks", Proc. EWSN 2004, Berlin, Germany, Jan. 2004.

13. Kappler and G. Riegel, "A Real-World, Simple Wireless Sensor Network for Monitoring Electrical Energy Consumption”, Proc. EWSN 2004, Berlin, Germany, Jan. 2004.

14. S. Antifakos, F. Michahelles, and B. Schiele, "Proactive Instructions for Furniture Assembly", Proc. Ubicomp 2002, Gothenburg, Sweden, Sept. 2002.

15. The 29 Palms Experiment: Tracking Vehicles with a UAV-Delivered Sensor Network. http://robotics.eecs.berkeley.edu/ pister/29Palms0103, Jan 2008

16. W. M. Meriall et al., "Collaborative Networking Requirements for Unattended Ground Sensor Systems", Proc. IEEE Aerospace Conf., Mar. 2003.

17. G. Simon, A. Ledezczi, and M. Maroti. "Sensor Network-Based Countersniper System", Proc. SenSys, Baltimore, MD, USA, Nov. 2004.

18. G. Gonçalves, A. Sousa, J. Pinto, P. Lebres, J. Borges de Sousa, "Pilot Experiment of an Early Warning Fire Detection System", $3{ }^{\text {rd }}$ European Workshop on Wireless Sensor Networks, Switzerland, February 2006.

19. Jan Rabaey, et al, "PicoRadio: Communication/Computation PicoNodes for Sensor Networks", Technical Report, Electronics Research Laboratory, pp. 7-22, U.C. Berkeley, Dec. 2002.

20. Cha, H., Choi, S., Jung, I., Kim, H., Shin, H., Yoo, J., and Yoon, C., "RETOS: resilient, expandable, and threaded operating system for wireless sensor networks", Proc. IPSN'07, Cambridge, Massachusetts, USA, April 2007, pp. 25 - 27

21. Bhatti, S., Carlson, J., Dai, H., Deng, J., Rose, J., Sheth, A., Shucker, B., Gruenwald, C., Torgerson, A., and Han, R. 2005, "MANTIS OS: an embedded multithreaded operating system for wireless micro sensor platforms" Mob. Netw. Appl. 10, 4 (Aug. 2005)

22. TinyOs, http://www.tinyos.net, Jan 2008

23. SOS, http://nesl.ee.ucla.edu/projects/sos, Jan 2008

24. Contiki, http://www.sics.se/contiki, Jan 2008

25. Vinícius C. de Almeida, Luiz Filipe M. Vieira, Breno A. D. Vitorino, Marcos Augusto M. Vieira, Diógenes C. da Silva Jr., Antônio O. Fernandes, Claudionor Nunes Coelho Jr., "Sistema Operacional YATOS para Redes de Sensores sem Fio", Workshop de Sistemas Operacionais 2004, Salvador/BA, Agosto 2004

26. P. Levis and D. Culler. Mate, "A tiny virtual machine for sensor networks. In International Conference on Architectural Support for Programming Languages and Operating Systems", San Jose, CA, USA, Oct. 2002

27. Y. Wang, S. H. Cho, C. G. Sodini, and A. P. Chandrakasan, "Energy Efficient Modulation and MAC for Asymmetric RF Microsensor Systems", Intl. Symp. On Low Power Electronics and Design (ISLPED '01), August 2001, pp. 96-99

28. Schurgers, O. Aberthorne, and M. B. Srivastava, "Modulation Scaling for Energy Aware Communication Systems", In Intl. Symp. on Low Power Electronics and Design (ISLPED '01), August 2001, pp. 96-99

29. Holger Karl, Andreas Willig: "A Short Survey Of Wireless Sensor Networks", Technical Report, Telecommunication Networks Group, Technische Universitt Berlin, 2003 
30. J. Elson and K. Römer, "Wireless sensor networks: a new regime for time synchronization", ACM SIGCOMM Computer Communication Review, 2003, pp. 149-154

31. Pavlos Papageorgiou, "Literature Survey on Wireless Sensor Networks", July 2003

32. C. Intanagonwiwat, R. Govindan, D. Estrin, J. Heidemann, and F. Silva. Directed Di fusion for Wireless Sensor Networking. IEEE Trans. on Networking, February 2003.

33. Chieh-Yih Wan, A.T. Campbell, and L. Krishnamurthy, "PSFQ: a reliable transport protocol for wireless sensor networks" ACM International Workshop on Wireless Sensor Networks and Applications, 2002

34. Jamal N. Al-Karaki and Ahmed E. Kamal, "Routing techniques in wireless sensor networks: a survey", IEEE Wireless Communications Journal, December 2004

35. Tinydb, http://telegraph.cs.berkeley.edu/tinydb/, Jan 2008

36. R. Avnur and J. M. Hellerstein, "Eddies : Continuously Adaptive Query Processing", Proc. 2000 ACM SIGMOD Intl. Conf. on Management of Data, Dallas, TX, May 2000, pp. 261-272

37. H. Gupta, S. Das, and Q. Gu., "Connected Sensor Cover: Self-Organization of Sensor Networks for Efficient Query Execution" Proc. 4th ACM Intl. Symp. on Mobile Ad Hoc Networking and Computing (MobiHoc), Annapolis, MD, 2003

38. N. Sadagopan, B. Krishnamachari, and A.Helmy, "The ACQUIRE mechanism for efficient querying in sensor networks" Proc. 1st IEEE Intl. Workshop on Sensor Network Protocols and Applications (SNPA), Anchorage, AK, May 2003. 